\title{
Dull Professional Data From Ordinary Precedents
}

\author{
FEDERICO GARCIA LAMMERS
}

South Dakota State University

This paper begins with an easily refuted assumption. Most formal architectural education culminates in two paths: the individual thesis project or the practice-based internship. The latter path is wrought with the practicalities of professional anticipation, while the former is bound by disciplinary expectations to articulate novel theories that often result in some instrumental application or goal. This paper focuses on a graduate studio that addresses how to end architectural education by combining profeional practice, precedent study, and speculative research. Since 2016, there have been twenty students, six professional practices, fifteen architects, and three faculty involved in the Forensics Studio at South Dakota State University.

The work from the studio is a forensic investigation into the decision-making and execution of an existing building. It operates like a detective story. Time simultaneously moves forward in the project time line and backward in the process of investigation. Where they meet is where motive is uncovered. In their last semester of study, teams of graduate students collaborate with one architecture firm to research the critical workflows of that practice. Practitioners facilitate the exchange of existing data, arrange site visits, and participate in studio reviews. Through the making of time-based images students explore professional networks by fetishizing ubiquitous and seemingly dull processes, such as, meeting minutes, field observations, specifications, emails, and more.

There is a great deal of "non-architecture" work performed by architects and introduced to students in professional practice courses. Many faculty and professionals remark with frustration, "I worked on emails, RFIs, specs, etc. I didn't design anything, I didn't do architecture today." What would happen if processes that are typically excluded from graphical representation had to be used to articulate the ideation and execution of a building? How can we unfold the complexity of ordinary architectural life in an academic context without trying to simulate architectural practice? What is the relevance of examining the theoretical underpinnings of dull professional processes, or "non-architecture work?"

\section{UNPOPULAR FORENSICS}

The origin of the word forensics comes from the Latin forensis, which refers to the forum, and thus to the practice and skill of making an argument before a professional, political or legal gathering. According to Eyal Weizman, architect and professor of spatial and visual cultures at the University of
London and director of Forensic Architecture, forensics is about science as an art of persuasion. ${ }^{1}$

The idea of forensics is present in popular culture through a wide range of films and television shows, living in the media based imagination of many people across the world. In professional terms, architectural forensics is a specialized and financially lucrative field that focuses on the legal implications of architectural work by using surveying techniques in the context of litigation. In spite of its place in popular culture and its advancement in professional fields, forensic methodologies are typically not explicitly folded into architectural education. A fundamental component of such methodologies is the role of prosopopoeia. Prosopopoeia is a rhetorical device used to endow an inanimate object or subject with a voice. ${ }^{2}$ The language of things (figure 1 ), and its translation is one of the essential forensic aspects of architecture.

To define the "language of things" it is necessary to describe forensics as a networked relationship among three things: a subject, a mediator, and a forum. In the graduate studio presented in this paper, students, faculty, and professionals are the mediators, the buildings that are being studied are the subjects, and the forum becomes the exchange between the studio space and the architecture offices. Each category is elastic, with the forum providing the technology with which the mediator's claims about the subject can be made. The forum is not only a given space, it is a framework produced through a series of entangled performances and connected networks. The traditional design studio starts with broad ambitions that are narrowed into a set of details supporting broader claims. Forensics starts from a set of narrow concerns-seemingly insignificant or too small to matter-that build outward networks to address large disciplinary questions. The questions that emerge in the forensics studio are based on the effects of electronic media, the investigation of collaborative practices, and the role of politics on the reconfiguration of architectural authorship.

Eyal Weizman asserts that forensics is not about the single subject in isolation, instead it is about the chains of associations that come from it and connect it to people, technologies, and ideas - it is the flexible network between people and things. ${ }^{3}$ Students begin and end the semester with a completed or soon to be completed building, an object in apparent isolation. To frame the student work, and to expand upon the language of the object, forensics is referred to in three ways: 
- Referring to the forum and the practice of making an argument before a professional or academic gathering

- Referring to the techniques used to develop investigative strategies and tactics

- Referring to the conception of time based on the nonlinear sequencing of events
These three criteria are synthesized into three steps that connect the studio schedule with its intellectual scope. The steps are not mutually exclusive, however, each step intersects with one or more of the three factors described at the beginning of the paper: professional practice, precedent study, and speculative research. The student work shown in this paper highlights that intersection.

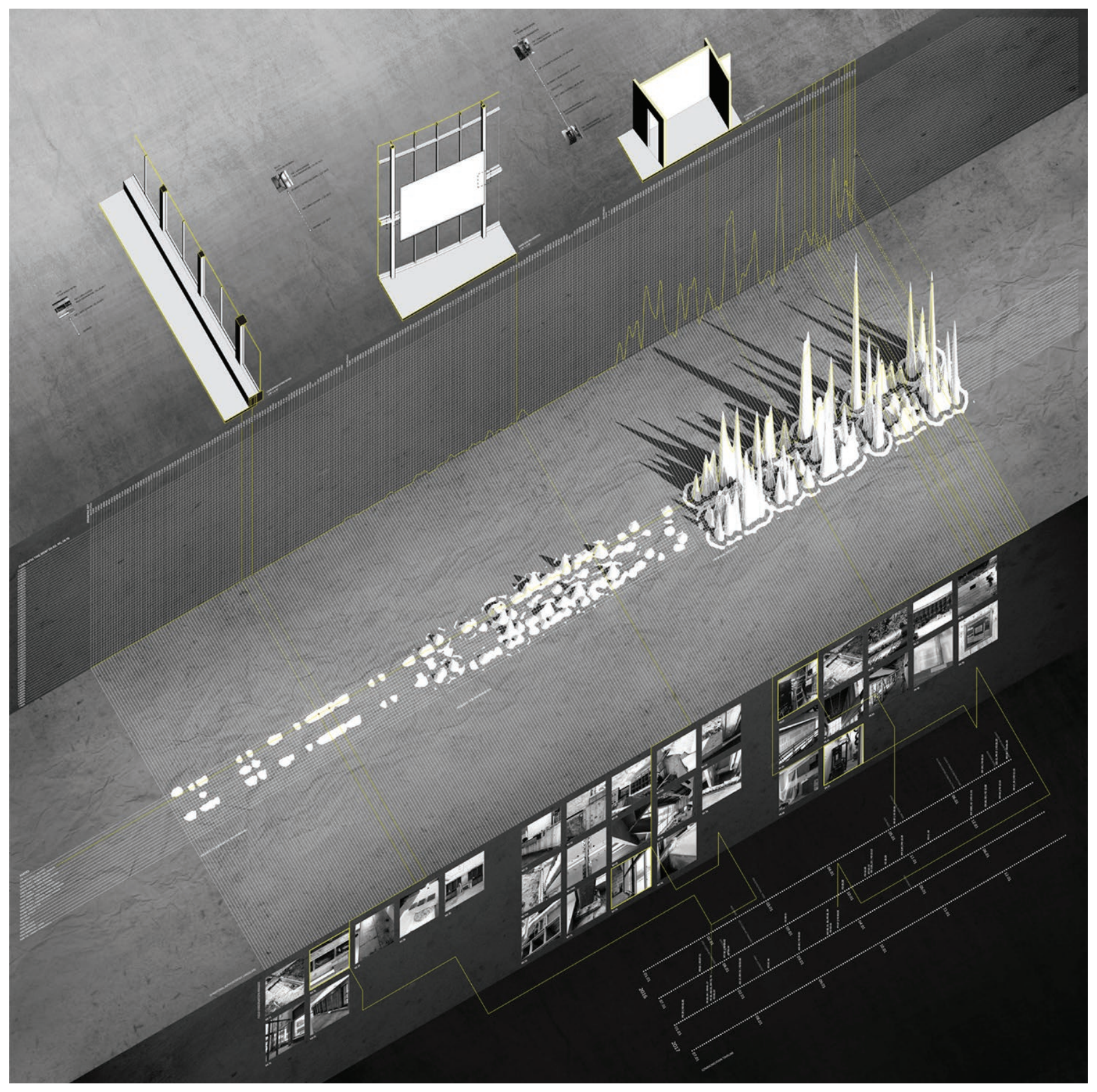

Figure 1. Time-based data landscape articulating the "language of things," based on three seemingly inconsequential building elements (columns, mullions, and IT room), which connect three forms of ubiquitous communication (1. Architect's supplemental information, 2. Requests for information, and 3. Proposal requests) to the displacement of on-site architectural authority. 
[CREATED]

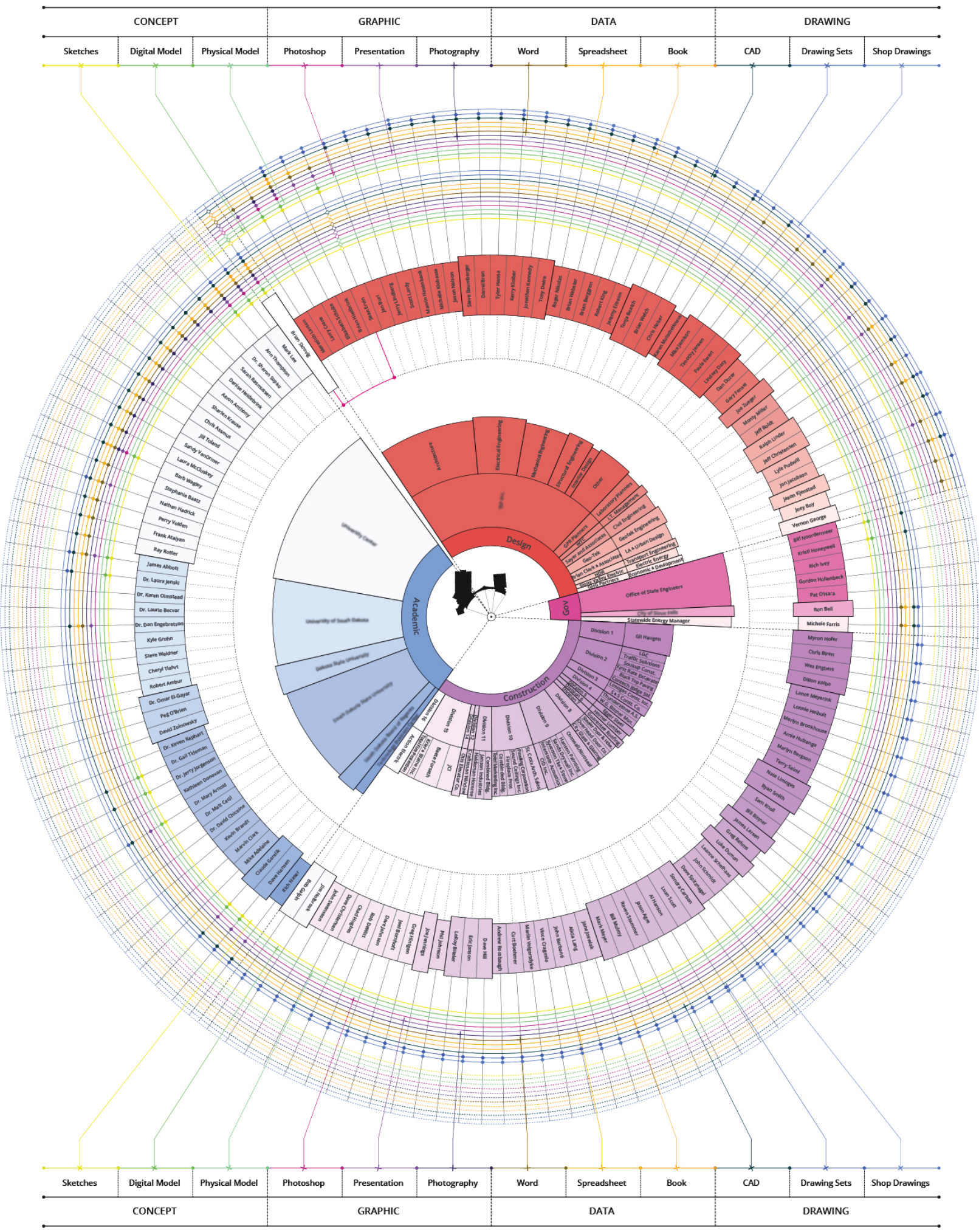

[RECEIVED] 


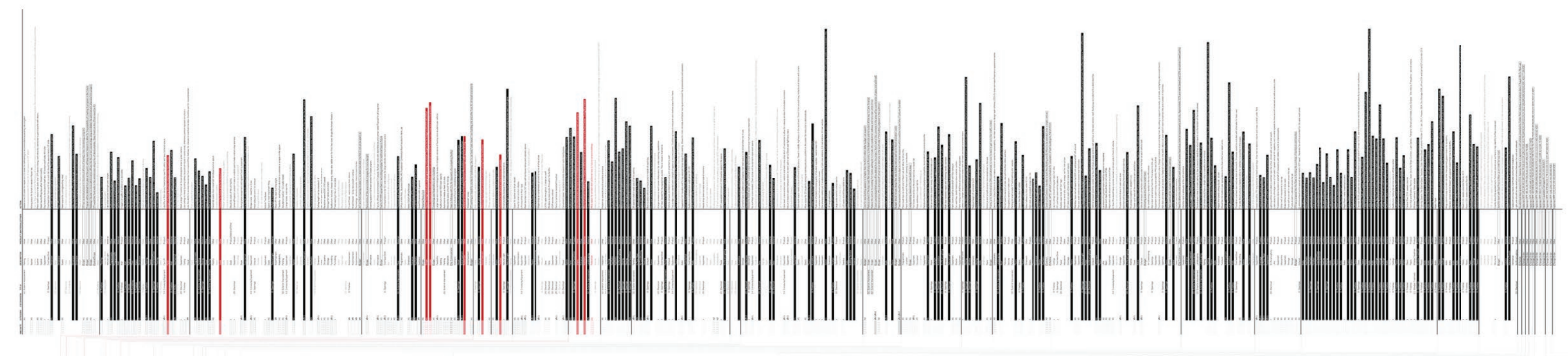

Figure 3: Four years of meeting minutes, specifications-based requests for information exchanged to develop a public education technology building. The project time-line is turned into a record of the effects of product and equipment selection in the context of building information modeling processes.

The red bars show the amount of time dedicated to the selection, re-selection and replacement of equipment and building systems, while the black bars indicate the meetings dedicated to finalize decisions.

\section{BUILDING STORIES}

The first step is an investigation of the building's effectsbasic functions and the relationships among owner, architect, financiers, and other building professionals. A portion of this work mirrors the traditional precedent study process. After being connected to the project's architects, engineers, contractors, and clients, students interrogate the situation and graphically dissect the building. The second step is connecting the facts of the project into a plausible story of how the project reached its end. In this phase, students are graphically mapping out webs of interconnectivity between people, tools, and place (figure 2). Webs are mapped by analyzing documents and data shared by the architecture firms. The third step is the graphical telling of each of these stories of a building process, and re-presenting the building to its progenitors. Where the crux of the story lies both structurally and stylistically is the critique. The critique is based on linking broad disciplinary questions to professional processes.

A great deal of "traditional" architectural labor is, on some level, discursive labor-and yet. The practice of architecture continues to find new purchases beyond (though often complementing) the making of buildings. ${ }^{4}$

\section{-James Graham, The Politics of Publication}

Benchmarks associated with the ideation and execution of a building define its early life. Specific document packages provide written and graphic evidence of the status, contract structure, and management of a project. Concept Design, Schematic Design, Design Development, Construction Documents, Construction Administration, these are the graphic and written forms of evidence that describe traditional practice and the steps necessary to coordinate design and construction. The time-frame changes, the delivery methods evolve, but benchmarks remain essential in the execution of most buildings and their respective contracts.
The images made after a building is complete, post occupancy evaluations measuring its efficiency, and articles written about its social relevance are often used as measures of the building's perceived success. These are metrics through which the practice of architecture and its results are measured and valued. This studio is interested in the interrogation of commonly used metrics in the early life of a building. The images of student work included in this paper do not question the intent of the architects who worked on each project. Instead, they prioritize and magnify minor effects-analogous to Miguel de Unamuno's Intrahistorias-emphasizing that impact and intent are two different things. ${ }^{5}$ The structure of each image is based on the linearity of the benchmarks mentioned previously. Supposed minor effects, such as the large amounts of communication recording the displacement of the IT room in figure 1 , or the four years of specification development and product selection documented in figure 3 , point to the ubiquity of work that exists outside of design intent and in parallel to the illusion of tidy linearity associated with traditional benchmarks.

What if we acknowledge that the benchmarks associated with the linearity of the design and construction process are not a faithful articulation of the most poignant moments in the story of the ideation and construction of a building? In fact, the moments of exceptions that are relevant to the narrative of a building are seldom documented and almost never articulated through graphic means-typically existing outside the scope of contracts.

Drawings, or more accurately, two-dimensional images output from computer models show no evidence of client disputes or political tensions surrounding a project (figure 4). In most cases, these documents reinforce their instrumental delivery role, even though their frozen two-dimensionality has not been instrumental in vast sections of the construction industry for over a decade. ${ }^{6}$ 
The graphics or benchmarks of an architectural project are the packaged synthesis, not the analytical exception of the work. Leveraging project specific exceptions against the practice of architecture is where emerging forms of practice emerge. Increasingly, these forms of practice probe at traditional methods of organizing people towards a common goal.

\section{COLLABORATIVE MYTHS}

It is easy to take for granted that architecture is a form of collective knowledge produced by groups of people across time. Because of its pervasiveness, collaboration is a misunderstood and under examined method of teaching architecture. Like forensics, the contemporary notion of collaboration permeates popular culture. The link between forensics and collaboration has normalized our perception of advanced technologies and connected them to people and professional processes without making important disciplinary links. The etymology and legacy of the word implies two things:

- Involvement in a subversive act

- Links between people and labor; Latin con ("with") and labōrō ("work")

In contemporary terms, there are two primary reasons why these definitions are problematic.

First, most collaboration no longer holds any subversive value. Its political content has been erased in favor of the amicable banding of people. This may seem positive, however, depoliticizing collaboration has led to benign trends of collaborative business models in many disciplines and professions. It is important for students to learn how to work with others. This is an inarguable fact that reinforces collaboration trends in academic and professional venues. If we can acknowledge that making architecture requires forms of collaboration - the process of iterative making and analysis-then why is it hard to problematize collaboration in architectural education?

Second, the relationship between architects and labor has been evolving since the Renaissance and is radically changing in the 21st century. As mentioned in the previous section, most design and construction documentation eliminates any record of conflict resulting from its production, which includes the inevitable collaborative tensions that emerge from multiple people working on a series of models and outputting images to establish alibis for these models.

In architecture today despite the proclaimed integration of all phases of the building process through high-tech management techniques, the rhetoric of immaterial production contributes to absolving architects from accountability to material bodies and places, not to mention provides an alibi from legal liability. ${ }^{7}$

-Joan Ockman, The Architect as Worker
Group projects and team efforts are common in the design studio. These efforts are usually driven by divisions of labor or preliminary work, such as, precedent analysis, shared site model construction, or other conceptually inspired exercises. Additionally, the studio is seen as a collaborative endeavour by virtue of positioning student work in physical (studio space) and conceptual (studio scope) proximity. Public critiques of student work reinforce this proximity. There is nothing wrong with these two approaches, however, very often they fail to problematize the role of collaboration. Architectural education's relationship to collaboration may only further its inevitable link to modes of commodification. What do we teach when there is nothing left but the calculable professional value of collaborative practices? ${ }^{8}$

Historic modes of collaboration seen in the pedagogical models of the Beaux Arts, Polytechnic, Bauhaus, and others, are one of the critical factors affecting the evolution of studio teaching in architectural education. ${ }^{9}$ This paper does not unfold each of these historic models, but rather acknowledges that there are increased levels of professionalization that continually place pressure on academic institutions to prepare students for the socio-technical aspects of daily work. These dull tasks are usually excluded from academic discourse and live outside the realm of design proficiency. They find a home in the purgatory of management practices and other "non-architecture work."

The collaborative aspects of architecture are being leveraged through the use of technology that allows groups of people to simultaneously access and share information to perform many dull tasks. The conflation between information and knowledge, or "the rhetoric of immaterial production," as Joan Ockman points out, has contributed to the distance between ideation and execution. The work presented in this paper examines the intellectual labor of designing buildings to understand and graphically interrogate the role of collaboration in professional practice.

In order to frame this dialog, the studio considers two distinct forms of collaboration: diachronic and synchronic. Synchronic collaborations connect people working in the same moment towards a common goal, often resulting in a single thing. Diachronic collaborations connect design attempts forming relationships that emerge across time. This type of collaboration can connect distant and distinct objects across space. ${ }^{10}$ At the beginning of each semester, all of the previous year's work is shared with the current cohort of students. This includes electronic files and books synthesizing each team's research. In some cases, students have decided to continue the work of their peers directly by studying the same building. In such instances, the diachronic nature of the work is literal. Whether they decide to expand upon the work of the peers directly, or continue to unfold questions posed in previous version of the studio indirectly, each version of the studio has 


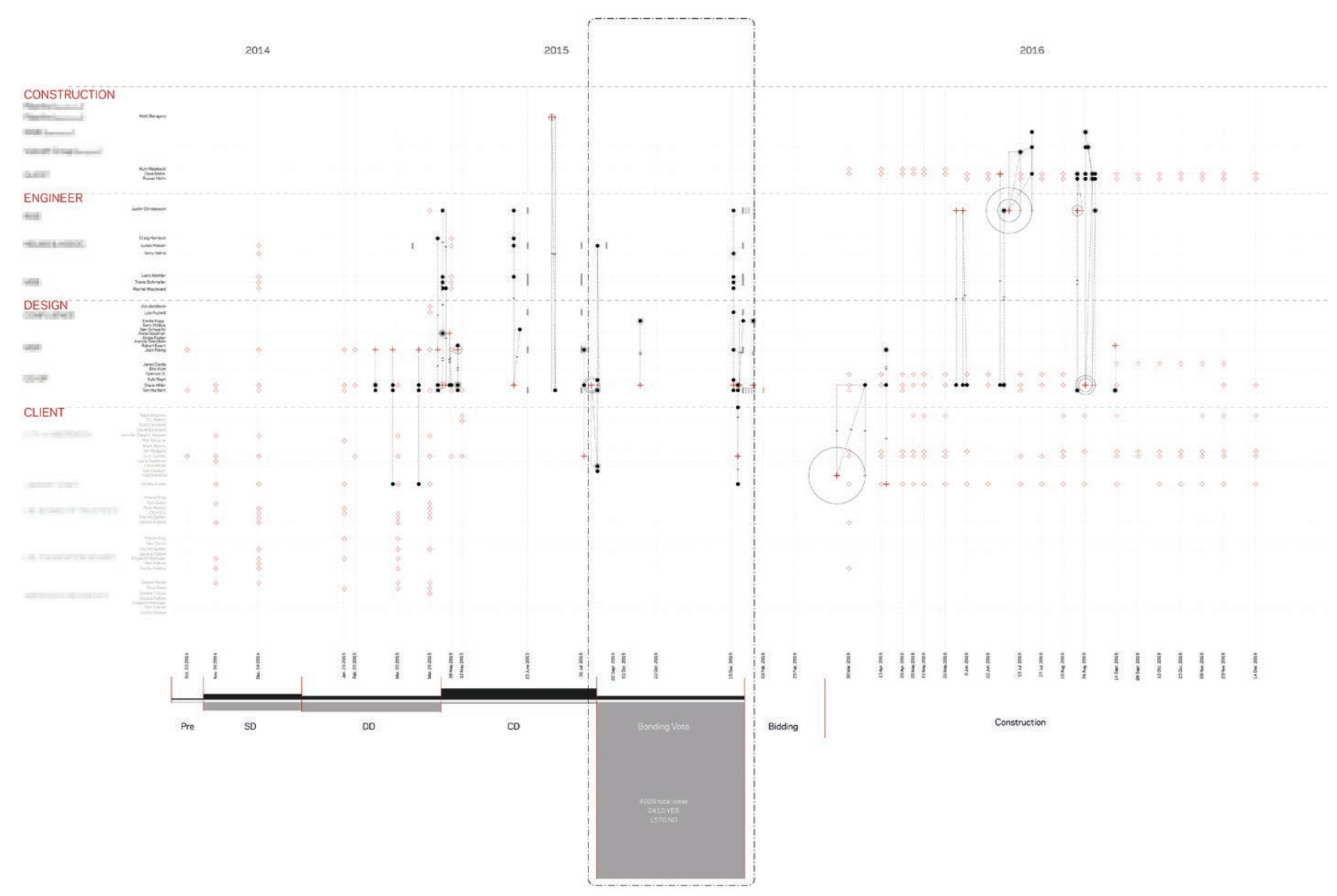

Figure 4: Two years of emails exchanges between building professionals and clients, recording the politics and referendum (grey block) of a public library project. Email is a ubiquitous form of communication, tacitly underlying the formation of knowledge associated with architecture. This is a notational system, which visualizes conversations between people to highlight the fragile public dimension of architectural processes.

resulted in a collection of work that blurs the singular authorship of the design studio and places the ongoing pursuit of everyday tasks at the center of the studio work.

In the Forensics Studio studio, the distinction between synchronic and diachronic forms of collaboration connects student work across different semesters and reinforces the relationship between the academic study of buildings and the professionals involved in making them. This type of collaboration hinges on the ability to theorize about professional work, instead of professionalizing theoretical work.

\section{ORDINARY MIMESIS}

The mimetic process-digesting and productively imitating-of precedent building analysis is fundamentally different when it is conducted via electronic media. In many professional and teaching contexts, the role of precedents has expanded to include processes tied to other disciplines, which unfold specific material performance, socially-driven interests, etc. The inspiration gleaned from building precedents, which is different from mimetic copying, still comes from an expanding cannon of seminal buildings across the world. In the Forensics Studio, the precedents are ordinary, everyday buildings that mark the pedagogical importance of often unrecognized architectural work. The effect is to make the practice of architecture in South Dakota the direct subject of the studio. Studying these ordinary buildings results in a series of data-driven landscapes and images that articulate the decisions affecting the local practice of architecture. In a technical age of expansive communication tools, these professional data landscapes highlight disciplinary questions at the intersection of architectural authority and collaboration. The Forensics Studio is building a critical lens through which to end students' education at this ordinary intersection.

Acknowledgements: The work produced in figures 1-4 is from the Forensics Studio in the Department of Architecture (DoArch) at South Dakota State University conducted from 2016 to 2018. The Forensics Studio is the final studio in the professional Master of Architecture curriculum at DoArch. Figure list and authors: Nurnberger, Jared and Yusuff, Quadri; Skrovig, Brian; Davis, Justin and Pfeil, Levi; and Millar, Ethan. 


\section{ENDNOTES}

1 Eyal Weizman, "Forensic Architecture," in Forensis, The Architecture of Public Truth (Berlin: Steinberg Press, 2014), 9.

2 Weizman, 16.

3 Eyal Weizman and Thomas Keenan, Mengele's Skull, The Advent of Forensic Aesthetics (Berlin: Steinberg Press, 2012), 25.

4 James Graham, "The Politics of Publication," in Asymmetric Labors: The Economy of Architecture in Theory and Practice (New York: The Architecture Lobby, 2016), 79.

5 Carlos Medina, "Intrahistoria, Cotidianidad y Localidad," Atenea 500 (2009): 125.

6 John May, "Everything is Already an Image" Log 40 (2017): 12.

7 Joan Ockman, "Foreword," in The Architect as Worker: Immaterial Labor, the Creative Class, and the Politics of Design, ed., Peggy Deamer (New York: Bloomsbury, 2015), xxiv.

8 Canedo Diaz Aravena and F. Toti, "Commodifying Architectural Education," in Asymmetric Labors: The Economy of Architecture in Theory and Practice (New York: The Architecture Lobby, 2016), 137.

9 Joan Ockman, Architecture School: Three Centuries of Educating Architects in North America (Cambridge, MA: The MIT Press, 2012), 111.

10 Andrea Zanderigo, "Editorial," in San Rocco: Collaborations, ed., Matteo Ghidoni (Perugia, Italy: Publistampa Arti Grafiche, 2013), 3. 